Editorial

\title{
Sarcopenia Should Reflect the Contribution of Age-Associated Changes in Skeletal Muscle to Risk of Morbidity and Mortality in Elderly People
}

\author{
William J. Evans $\mathrm{PhD}^{\mathrm{a}, \mathrm{b}, *}$ \\ ${ }^{a}$ KineMed, Inc, Emeryville, CA \\ ${ }^{\mathrm{b}}$ Division of Geriatrics, Duke Medical Center, Durham, NC
}

The loss of skeletal muscle mass with advancing age is a universal phenomenon. The trajectory of this loss of muscle is remarkably variable and its etiology complex and multifactorial. The term, sarcopenia, was initially described as the age-associated decrease in skeletal muscle mass and was thought to be similar to the phenomenon of osteopenia, the age associated loss of bone density that is predictive of an increase in risk of a bone fracture. ${ }^{1,2}$ The loss of muscle mass was thought to be the primary cause of decreased strength and functional capacity and a predictor of risk of disability or other outcomes. Indeed, a cross-sectional study with a wide range of ages from my laboratory seemed to confirm this idea, when we found that there were no significant age-associated differences in muscle strength when strength was expressed as a function of muscle mass. ${ }^{3}$ Frontera et $\mathrm{al}^{4}$ demonstrated that $90 \%$ of the loss in strength over 12 years was explained by decreased muscle cross-sectional area and initial muscle strength. However, a number of longitudinal studies have demonstrated that the loss of skeletal muscle mass and force production is not tightly linked, and among very old men and women, muscle size explains only $6 \%$ of the variability in muscle strength. ${ }^{5}$ In general, there is a greater loss of muscle strength than mass and weakness appears to be more closely associated with risk of disability and mortality. ${ }^{6}$ However, there are a number of ageassociated changes in many properties of skeletal muscle that may contribute to morbidity and mortality. ${ }^{7}$ Skeletal muscle is the primary site of glucose disposal and resistance of glucose transport into muscle is strongly associated with risk of type 2 diabetes along with a sequelae of conditions including microvascular disease, neuropathy, nephropathy, and eye damage (including cataract). Decreased muscle mass is the largest contributor to the age- associated decrease in basal metabolic rate, ${ }^{8}$ and the reduced energy requirement of elderly people to the well-characterized increased body fatness in elderly people. The broad range of metabolic and contractile changes that occur with advancing age and their contribution not only to weakness but to many of the diseases of aging should cause a rethinking of the term sarcopenia and what components of muscle function and metabolism should be measured to assess morbidity and mortality ${ }^{9}$ and how better to define sarcopenia.

DOI of original article: http://dx.doi.org/10.1016/j.jamda.2015.02.006

* Address correspondence to William J. Evans PhD, KineMed, Inc, 5980 Horton Street, Suite 470, Emeryville, CA 94608.

E-mail address: WEvans@KineMed.com.
While there is little dispute that loss of muscle mass with advancing age, sarcopenia, is a universal phenomenon, one of the central questions that remains controversial is this: when does an individual become sarcopenic? As new pharmacologic treatments with the potential to reverse muscle loss advance, the determination of who should be treated for the condition of sarcopenia is not straightforward. This controversy stems from the fact that amount of the loss of muscle mass does not always correspond to an equivalent reduction in muscle strength or function. Because muscle weakness is more closely associated with risk of disability than amount of muscle, the term dynapenia ${ }^{10}$ has been used to describe the loss of strength with advancing age. This has prompted consensus groups attempting to address this question to describe the condition of sarcopenia as a patient with low muscle function and mass. This definition would allow clinicians to identify patients with low functional capacity because of loss of muscle for treatment. The research by Hirani et $\mathrm{al}^{11}$ helps to more firmly establish the risk of sarcopenia as defined by different consensus groups. The study examined the risk associated with 3 different definitions of sarcopenia: sarcopenia I was characterized as low muscle mass only; sarcopenia II, low muscle mass and weakness; and sarcopenia III, low muscle mass, weakness, and slow gait speed. As expected, sarcopenia III (weakness, slow gait speed, and low muscle mass) was most strongly associated with increased risk of disability. This increased risk compared with sarcopenia I or II is likely due to the fact that slow gait speed ${ }^{12}$ and weakness ${ }^{13}$ have been independently associated with increased risk even when not considered with low amount of muscle. Importantly, this study ${ }^{11}$ also reports that sarcopenia I, II, and III are associated with increased risk of institutionalization and mortality, and the adjusted risks for institutionalization and mortality are significant for sarcopenia I and not dramatically increased by weakness and/or slow gait speed. This demonstrates the independent effects that low muscle mass alone confers on these risk factors. Appendicular lean mass was assessed using dual-energy X-ray absorptiometry. Age is associated with increased intramyocellular fat ${ }^{14}$ and, as a result, the amount of functional muscle in elderly people is likely significantly overestimated by dual-energy X-ray absorptiometry, which may lead to an underestimate of true risk of sarcopenia I.

This study has some important limitations that are pointed out by the authors. Women were not part of this study and the data for appendicular lean mass, strength, and gait speed were not 
longitudinal. The increased prevalence of all classifications of sarcopenia with increasing age strongly indicates increased severity with advancing age and without knowledge of the changes in muscle mass or function, the causes of variability in risk with advancing age remain unknown. Skeletal muscle is one of the most dynamic tissues in the human body. It adapts to changes in level of physical activity, diet, endocrine function, and more. The study of Hirani et $\mathrm{al}^{11}$ provides an important assessment of the considerable risk of loss of muscle mass and contractile function in men. Perhaps a more comprehensive definition of sarcopenia to include the primary role of skeletal muscle in determination of age-associated morbidity and mortality risk should also include insulin resistance and basal metabolic rate. Both additional factors can be easily assessed with a fasting blood sample (homeostatic model assessment) and an estimate of basal metabolic rate (from Harris-Benedict equation). These additional measurements along with an accurate measure of muscle mass and strength should be components of the definition of sarcopenia and could provide an index of the actual contribution of skeletal muscle to age associated risk to morbidity and mortality.

\section{References}

1. Evans WJ, Campbell WW. Sarcopenia and age-related changes in body composition and functional capacity. J Nutr 1993;123:465-468.

2. Evans WJ. What is sarcopenia? J Gerontol A Biol Sci Med Sci 1995;50:5-8.
3. Frontera WR, Hughes VA, Lutz KJ, Evans WJ. A cross-sectional study of muscle strength and mass in 45- to 78-yr-old men and women. J Appl Physiol (1985) 1991;71:644-650.

4. Frontera WR, Hughes VA, Fielding RA, et al. Aging of skeletal muscle: A 12-year longitudinal study. J Appl Physiol (1985) 2000;88:1321-1326.

5. Fiatarone MA, O'Neill EF, Ryan ND, et al. Exercise training and nutritional supplementation for physical frailty in very elderly people. N Engl J Med 1994; 330:1769-1775.

6. Newman AB, Kupelian V, Visser M, et al. Strength, but not muscle mass, is associated with mortality in the health, aging and body composition study cohort. J Gerontol A Biol Sci Med Sci 2006;61:72-77.

7. Wolfe RR. The underappreciated role of muscle in health and disease. Am J Clin Nutr 2006;84:475-482.

8. Tzankoff SP, Norris AH. Longitudinal changes in basal metabolic rate in man. J Appl Physiol 1978;33:536-539.

9. Janssen I, Ross R. Linking age-related changes in skeletal muscle mass and composition with metabolism and disease. J Nutr Health Aging 2005;9: 408-419.

10. Clark BC, Manini TM. Sarcopenia $=/=$ dynapenia. J Gerontol A Biol Sci Med Sci 2008;63:829-834.

11. Hirani V, Blyth F, Naganathan V, et al. Sarcopenia is associated with incident disability, institutionalization, and mortality in community-dwellling older men: The concord health and ageing in men project. JAMDA 2015;16:xxx.

12. Guralnik JM, Ferrucci L, Pieper CF, et al. Lower extremity function and subsequent disability: Consistency across studies, predictive models, and value of gait speed alone compared with the short physical performance battery. J Gerontol A Biol Sci Med Sci 2000;55:M221-M231.

13. Hicks GE, Shardell M, Alley DE, et al. Absolute strength and loss of strength as predictors of mobility decline in older adults: The InCHIANTI study. J Gerontol A Biol Sci Med Sci 2011;67:66-73.

14. Goodpaster BH, Kelley DE, Thaete FL, et al. Skeletal muscle attenuation determined by computed tomography is associated with skeletal muscle lipid content. J Appl Physiol (1985) 2000;89:104-110. 